

\title{
Machine Detector Interface for FASER
}

\author{
Helena Lefebvre ${ }^{\dagger},{ }^{*}$ S. Gibson and L. Nevay \\ John Adams Institute, Royal Holloway, University of London \\ Egham, UK \\ E-mail: helena.lefebvre.2017@live.rhul.ac.uk
}

The ForwArd Search ExpeRiment (FASER) is a planned small-scale experiment designed for the search of light and weakly-interacting particles at the Large Hadron Collider (LHC). The FASER detector will be located along the beam collision axis, $480 \mathrm{~m}$ downstream from the ATLAS interaction point (IP), in an unused service tunnel. A full Geant4 model of the LHC accelerator as well as the surrounding tunnel complex has been developed to simulate the propagation of the products of the proton-proton collisions from the ATLAS IP to the FASER detector. The simulation also handles radiation from LHC beam induced background sources. The simulated fluences of Standard Model particles, which constitute the main signal and background of the experiment are presented.

40th International Conference on High Energy physics - ICHEP2020

July 28 - August 6, 2020

Prague, Czech Republic (virtual meeting)

\footnotetext{
${ }^{\dagger}$ on behalf of the FASER collaboration

* Speaker
} 


\section{Introduction}

FASER is a planned small-scale and cost-effective experiment optimized for detection of light and weakly-interacting particles, such as neutrinos and hypothetical Beyond Standard Model (BSM) particles that could be produced during LHC collisions. FASER will be located along the beam collision axis, $480 \mathrm{~m}$ downstream from the ATLAS experiment [1], in an unused service tunnel [2]. FASER will be sensitive to long-lived particles produced along the beam collision axis, travelling hundreds of meters before decaying within the cylindrical detector volume, with radius $R=10 \mathrm{~cm}$ and length $\mathrm{L}=1.5 \mathrm{~m}$. FASER will significantly expand the LHC's discovery potential of light BSM particles. FASER is being installed to collect $150 \mathrm{fb}^{-1}$ of LHC collision data starting in 2022.

\section{Simulation Architecture}

Proton-proton collisions were simulated using the SIBYILL model in the Cosmic Ray Monte Carlo (CRMC) event generator [3]. Generated particles are propagated towards FASER using the Beam Delivery SIMulation (BDSIM) particle tracking code [4] capable of generating a Geant4 model [5] and tracking particles through an accelerator lattice and surrounding material. Particles reaching the FASER interface plane (IP, a virtual plane $1 \mathrm{~m}^{2}$ in front of FASER detector) are passed to FASER detector software for reconstruction and analysis.

\section{Simulation Model}

The FASER detector is located in an adjacent service tunnel TI12 and is approximately $5 \mathrm{~m}$ from the LHC tunnel. Weakly-interacting particles originating from ATLAS, initially travel through the LHC beam pipe for $140 \mathrm{~m}$ before interacting with an absorber, specifically designed to stop physics debris reaching the cold section of the accelerator. From the absorber, particles travel through the tunnel and surrounding materials outside of the beam pipe, than the tunnel wall, soil material, second tunnel wall and finally into the FASER detector. A model of the tunnel section between the ATLAS and FASER detectors was prepared by combining existing FLUKA models, a generic BDSIM tunnel section and a custom tunnel section created in pyg4ometry (fig. 1).

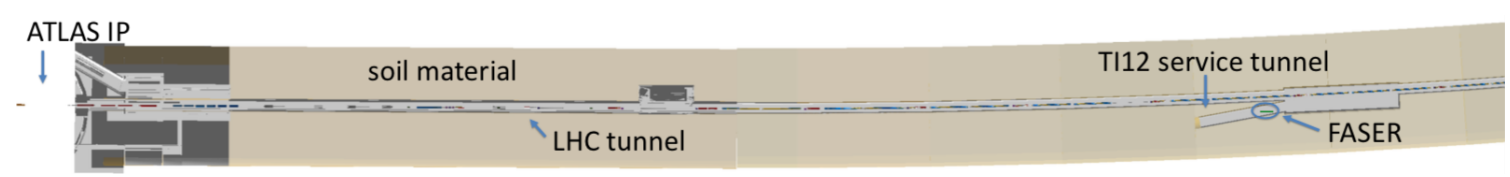

Figure 1: Model of tunnel section between ATLAS and FASER detectors.

\section{Results}

The detector response to Standard Model particles propagating to FASER including natural radiation background must be simulated to estimate the signal to noise ratio of Beyond Standard Model (BSM) searches. The main SM contribution originates from muons and neutrinos, whose fluxes are shown in Fig. 2, alongside the energy spectra of all particles reaching the detector. Muons and neutrinos are the only SM particles with a non-negligible flux at the FASER interface plane. 

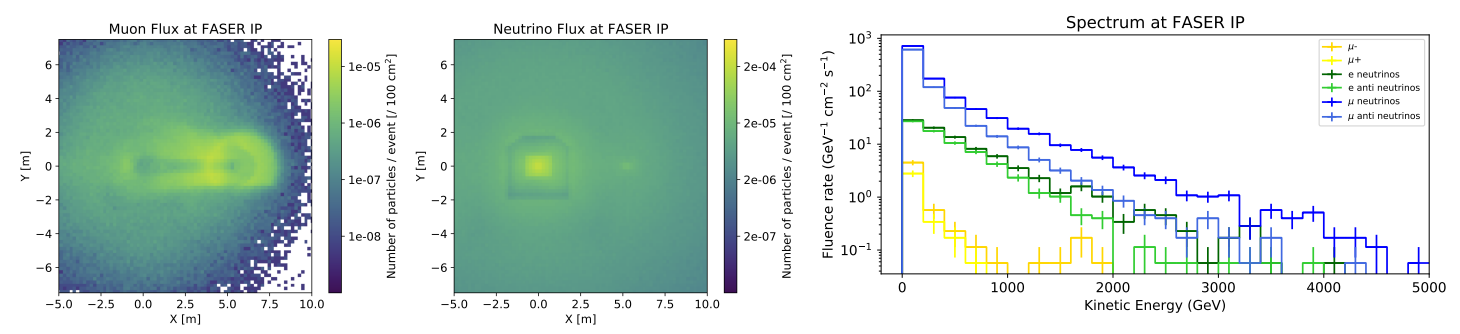

Figure 2: Muon (left) and neutrino (centre) fluxes at a $15 \times 15 \mathrm{~m}$ plane at FASER IP (FASER is located at $(0,0)$ coordinates.) Energy spectrum of particles reaching the FASER detector (right).

The muon flux is lower at the location of FASER, which is important, as muons are the background for neutrinos and BSM particles. Contrary to muons, the neutrino flux reaches a maximum at the FASER location. The energy spectrum plot shows that the muon neutrino signal, the dominant particle type reaching FASER, is larger by a factor 10 than electron neutrinos and by a factor of 100 than muons. Tau neutrinos were ignored in this analysis. The total number of particles reaching the FASER detector is $3.93 \times 10^{7} \pm 1.76 \times 10^{3}$ (stat.) $\mathrm{cm}^{-2} \mathrm{fb}$ (normalized to Run 3 parameters).

Different models are being explored to understand the effects of using different parameters such as event generators, the crossing angle of incoming colliding protons at the ATLAS IP, and geometry effects on the number of particles reaching the FASER detector.

\section{Summary}

The FASER detector will search for beyond Standard Model, light weakly-interacting BSM particles and study the interactions of high-energy neutrinos. A model of particle propagation was prepared using BDSIM to obtain the particle fluxes incoming at the FASER interface. The flux and energy spectra of particles reaching the detector (neutrinos and muons) were presented. Future work will evaluate the systematic uncertainty.

\section{References}

[1] G. Aad et al., "The ATLAS Experiment at the CERN Large Hadron Collider", JINST 3, S08003 (2008).

[2] A. Ariga et al., "FASER: ForwArd Search ExpeRiment at the LHC", [arXiv:1901.04468].

[3] T. Pierog, C. Baus, and R. Ulrich, CRMC, https://web.ikp.kit.edu/rulrich/crmc.html

[4] L. J. Nevay et al., "BDSIM: An accelerator tracking code with particle-matter interactions", Comput. Phys. Commun. 252 (2020).

[5] S. Agostinelli et al., "GEANT4-a simulation toolkit", Nucl. Instrum. Meth. A 506 (2003), 250-303, doi:10.1016/S0168-9002(03)01368-8. 